\title{
Hormônios
}

\section{Respostas hormonais e imunes agudas decorrentes do treinamento de força em Bi-Set}

\section{Denis Foschini',2 - CREF 017943-G/SP \\ denisfoschini@gmail.com}

\section{Jonato Prestes',3 - CREF 007176-G/PR}

jonatop@gmail.com
- Faculdade de Fisioterapia e Educação Física

2 Universidade Metodista de São Paulo/SP

${ }^{3}$ Faculdade de Ciências da Saúde

Foschini D, Prestes J. Respostas hormonais e imunes agudaes decorrentes do treinamento de força em Bi-Set. Fit Perf J 2007; $6(1): 38-44$.

RESUMO - O objetivo deste estudo foi investigar os efeitos do treinamento de força sobre as células do sistema imune, testosterona e cortisol mediante a alteração na ordem dos exercícios e no tempo de intervalo entre as séries. Foram estudados 9 homens com idade de 22,2 + 1,9 anos e experiência de 12 meses em treinamento de força. Foi realizado o método de múltiplas séries em bi-set. Foram coletadas amostras antes e imediatamente após os exercícios. Houve aumento no cortisol, leucócitos totais, neutrófilos, linfócitos e monócitos. A concentração de testosterona e a razão testosterona/cortisol não sofreram alterações. A mudança na ordem dos exercícios e no tempo de intervalo alteraram a resposta das células imunes e aumentaram o cortisol, constituindo-se um estímulo diferenciado para os indivíduos.

Palavras-chave: Treinamento de força, sistema imune, hormônios.

Endereço para correspondência:

Rua Luis da Silva Leite, 85, Vila Formosa, São Paulo, SP, CEP 0.3377-070.

Data de Recebimento: Setembro / 2006

Data de Aprovação: Novembro / 2006

Copyright@ 2006 por Colégio Brasileiro de Atividade Física Saúde e Esporte. 


\section{ABSTRACT}

Acute hormonal and immune responses after a bi-set strength training

The purpose of this study was to investigate the effects of strength training on immune cells, testosterone and cortisol by the alteration in the order of the exercises and rest interval. Nine males were accomplished, with $22.2 \pm 1.9$ years old and a minimal experience of 12 months in strength training. The multiples sets and bi-set method were accomplished. The samples were collected before and immediately after exercise. There was a significant increase in cortisol levels, total leukocytes, neutrophils, lymphocytes and monocytes. There were no alterations in testosterone and testosterone/cortisol ratio. The alteration in the exercise order and duration of interval modified the immune cells response and increased cortisol levels, constituting a differentiated stimulus for the individuals.

Keywords: Strength training, immune system, hormones.
Respuestas hormonales e immunes agudas resultantes del entrenamiento de fuerza en bi-set

El objetivo de este estudio fue investigar los efectos del entrenamiento de fuerza sobre las células inmunes, testosterona y cortisol mediante la alteración en la orden de los ejercicios y tiempo de intervalo. Fueron estudiados 9 hombres con edad de 22,2 $\pm 1,9$ años y experiencia mínima de 12 meses en entrenamiento de fuerza. Fue realizado el método de múltiples series en bi-set. Fueron recolectadas muestras antes y después de los ejercicios. Hubo aumento en el cortisol, leucocitos totales, neutrofilos, linfocitos y monocitos. La testosterona y la razón testosterona/cortisol no sufrieron alteraciones. La alteración en la orden de los ejercicios y tiempo de intervalo alteraran la respuesta de las células inmunes y del cortisol, constituyéndose un estímulo diferenciado para los individuos.

Palabras-clave: Entrenamiento de fuerza, sistema inmune, hormonas

\section{INTRODUÇÃO}

Os mecanismos e efeitos adaptativos, especialmente hormonais e imunológicos do treinamento de força ainda precisam ser sistematicamente elucidados. Este tipo de exercício é dependente da variação de alguns fatores: o movimento específico do exercício, a carga utilizada, a duração das repetições, intervalo entre as séries e exercícios, tipo e velocidade da ação muscular e ordem dos exercícios. Diferenças na duração do intervalo de recuperação entre as séries influenciam a resposta neuroendócrina e imunológica ao treinamento de força ${ }^{1}$. Mayhew et al. ${ }^{2}$ analisaram praticantes de treinamento de força que realizaram uma sessão de 10 séries com 10 repetições a $65 \%$ de 1 RM no exercício leg press, utilizando um intervalo de recuperação de 1 minuto e 3 minutos entre as séries. Os autores verificaram que intervalos de recuperação mais curtos promoveram leucocitose mais pronunciada e maior elevação na contagem de linfócitos, monócitos e neutrófilos circulantes, quando comparados com intervalos mais prolongados. $\bigcirc$ treinamento de força com curtos intervalos de recuperação (60 segundos) induz aumentos mais significativos nas catecolaminas, cortisol, testosterona e hormônio do crescimento $(G H)$, que são agentes causadores da redistribuição dos leucócitos durante e após o exercício ${ }^{3}$.

Sessões de treinamento com suficiente intensidade e volume podem elevar a reposta aguda da testosterona e do cortisol ${ }^{4}$. Schwab et al. ${ }^{5}$ registraram elevações significativas na testosterona frente a dois protocolos de agachamento. No entanto, Ahtiainen et al. ${ }^{6}$ não encontraram diferenças na resposta aguda da testosterona entre dois protocolos com número de repetições similares, porém com maior intensidade em um deles, que incorporou repetições forçadas; no entanto, o cortisol apresentou maior elevação no protocolo mais intenso. Foi demonstrado que 6 séries de 10RM no agachamento com 2 minutos de intervalo aumentaram significativamente as concentrações séricas de cortisol ${ }^{7}$. Diferentemente da testosterona, que exerce efeitos anabólicos, como o aumento da síntese protéica, o cortisol exerce funções católicas, como a lipólise, degradação protéica e redução da síntese protéica nas células musculares ${ }^{8}$.

Neste sentido, poucos trabalhos investigaram a resposta hormonal e imunológica pela alteração na ordem dos exercícios ou pela realização de exercícios em bi-set (dois exercícios em seqüência sem descanso). Sendo assim, o objetivo deste estudo foi analisar o efeito agudo de uma sessão de treinamento de força sobre as células do sistema imune, cortisol e testosterona, alterando a ordem dos exercícios, tipo e tempo de intervalo entre as séries em indivíduos treinados.

\section{MATERIAIS E MÉTODOS}

\section{Seleçã॰ dos Sujeitos}

critério adotado para inclusão dos voluntários ao estudo teve como pré-requisitos: ser do sexo masculino, experiência mínima de 12 meses em treinamento de força, realizando o protocolo com objetivo de hipertrofia muscular (Intensidade entre 6 e 12 repetições máximas) e estar utilizando os mesmos exercícios selecionados para o estudo, porém em ordem diferente (Tabela 1). Foram excluídos os indivíduos com história conhecida de doença cardiovascular, respiratória, diabetes, hipertensão, desordem hormonal, lesão muscular (últimos 12 meses), além daqueles que estavam administrando ou haviam administrado medicação ou suplementos nos 6 meses que antecederam o início do estudo. Contudo, foram selecionados 9 indivíduos aparentemente saudáveis. As características antropométricas dos participantes estão apresentadas na Tabela 1.

experimento foi aprovado pelo Comitê de Ética em Pesquisa 
Tabela 1

Características antropométricas dos voluntários ( $\mathrm{N}=9$ )

\begin{tabular}{lrrrr}
\hline Variáveis & $\mathrm{X}$ & $\mathrm{DP}$ & $\min$ & máx \\
\hline Massa corporal total $(\mathrm{Kg})$ & 77,8 & 12,3 & 60,6 & 106,5 \\
Estatura (cm) & 175,9 & 5,5 & 165,0 & 185,0 \\
Idade (anos) & 22,2 & 1,9 & 20,0 & 25,0 \\
\% de gordura & 10,6 & 4,7 & 4,7 & 19,1 \\
Massa gorda (Kg) & 8,9 & 5,2 & 2,8 & 20,4 \\
Massa magra (Kg) & 69,8 & 8,0 & 57,8 & 86,1 \\
\hline
\end{tabular}

Os valores estão expressos em média+DP. Média amostral (X), desvio padrão (dp), valor mínimo (min) e valor máximo (máx)

da Universidade Metodista de São Paulo - CEP - UMESP (Parecer no 074709/05), estando de acordo com a declaração de Helsinki.

Todos os participantes foram informados detalhadamente sobre os procedimentos utilizados e concordaram em participar de maneira voluntária do estudo, assinando termo de consentimento informado.

\section{Recomendações nutricionais}

Os participantes do estudo receberam um café da manhã individualizado, de acordo com a sua composição corporal, orientado por um profissional habilitado e qualificado, sendo oferecidos os macronutrientes pela quantidade em $\mathrm{g} / \mathrm{Kg}$ nas porcentagens de macronutrientes estabelecidas pelo American College of Sports Medicine e Dietitians Canada Joint Position Statement?.

\section{Teste de força máxima (1RM)}

Um dia após as avaliações antropométricas, foram realizados os testes de 1 repetição máxima (1RM). Após o aquecimento geral (10 minutos de caminhada em esteira rolante), os indivíduos executaram uma série de aquecimento específico de oito repetições a aproximadamente $50 \%$ da 1 -RM estimada (de acordo com a carga que os participantes realizavam antes do início do estudo).

Em seguida por outra série de três repetições a 70\% da 1-RM estimada para os primeiros exercícios da seqüência (fly com halteres e rosca martelo), o teste foi realizado para todos os exercícios, seguindo a mesma ordem da sessão de treinamento (fly inclinado com halteres, rosca martelo, supino inclinado, rosca scott, supino reto e rosca direta).

Os levantamentos subseqüentes foram repetições simples com cargas progressivamente mais pesadas. Repetiu-se o teste até que a 1-RM fosse determinada. $O$ intervalo de descanso entre as séries foi de três minutos e o número de tentativas para determinação da carga máxima foi de três, seguindo as descrições de Matuszak et al. ${ }^{10}$.

Todos os procedimentos para determinação da força máxima dinâmica, inclusive a padronização das angulações de movimentos seguiram as descrições de Brown e Weirr' ${ }^{11}$.

Foram selecionados 2 exercícios por dia, sendo estas sessões de teste separadas por 48h, para evitar influências na determinação das cargas máximas (na semana dos testes os participantes não realizaram qualquer tipo de exercício físico).

\section{Protocolo de treinamento}

protocolo seguiu as recomendações do American College Sports Medicine ${ }^{12}$. $O$ treinamento de força com múltiplas séries (método que utiliza mais de uma série por grupo muscular) e em bi-set (consiste em realizar dois exercícios sem intervalo passivo "parado"). A sessão foi realizada $72 \mathrm{~h}$ após os testes de força. Foram realizados três exercícios para adução horizontal de ombros, e três exercícios para flexão de cotovelos. Todos os participantes utilizavam no seu programa de treinamento os exercícios selecionados para o protocolo, porém o método e a ordem dos exercícios eram diferentes.

Para adução horizontal de ombros os exercícios foram: supino reto, supino inclinado e fly inclinado com halteres e para flexão dos cotovelos os exercícios foram: rosca direta, Rosca scott e rosca martelo. Em todos os exercícios, foram realizadas 3 séries buscando o maior número possível de repetições a $70 \%$ da 1 RM determinada, individualmente, a partir do teste de força máxima. A Tabela 2 apresenta como foram combinados os exercícios, sendo os voluntários orientados a realizar cada combinação sem intervalo "passivo" (descanso total - "parado") entre as séries. Por exemplo, na combinação 1 foi realizado o fly inclinado com halteres, ao terminar, o indivíduo realizava a rosca martelo, em seguida voltava para o fly inclinado.

procedimento foi realizado 3 vezes em cada combinação e, então, o indivíduo se dirigia para a execução da próxima combinação. Esse procedimento foi adotado para as três combinações.

\section{TABELA 2}

COMBinaÇões dos eXercícios

\begin{tabular}{lcc}
\hline & $\begin{array}{c}\text { Adução horizontal } \\
\text { de ombro }\end{array}$ & $\begin{array}{c}\text { Flexão } \\
\text { de cotovelo }\end{array}$ \\
\hline Combinação 1 & Fly inclinado com halteres & Rosca martelo \\
Combinação 2 & Supino inclinado & Rosca scott \\
Combinação 3 & Supino Reto & Rosca direta \\
\hline
\end{tabular}


TABELA 3

Comparação da concentração de testosterona, cortisol e a razão t/C entre os tempos: "Antes" e "Após" A intervenção (N=9)

\begin{tabular}{lccccccc}
\hline Variáveis & \multicolumn{1}{c}{$\mathrm{X}$} & $\mathrm{dp}$ & $\mathrm{min}$ & $\mathrm{máx}$ & perc 25 & perc 50 & perc 75 \\
\hline Testosterona Antes (nmol/L) & 14,7 & 4,7 & 9,4 & 24,0 & 11,0 & 12,5 & 18,2 \\
Testosterona Após (nmol/L) & 16,5 & 5,2 & 6,5 & 22,8 & 13,6 & 16,1 & 21,9 \\
Cortisol Antes (nmol/L) & 343,2 & 115,5 & 148,9 & 508,9 & 259,5 & 340,8 & 442,1 \\
Cortisol Após (nmol/L) & $516,8^{*}$ & 181,6 & 216,7 & 750,6 & 411,0 & 456,4 & 719,9 \\
\hline Razão T/C Antes & 0,048 & 0,026 & 0,025 & 0,104 & 0,03 & 0,039 & 0,064 \\
Razão T/C Após & 0,035 & 0,017 & 0,016 & 0,071 & 0,021 & 0,031 & 0,044 \\
\hline
\end{tabular}

Os dados apresentados na tabela representam a média amostral (X), desvio padrão (dp), valor mínimo (min), valor máximo (máx), percentil 25, percentil 50 e percentil 75.

*Diferença estatisticamente significativa da concentração da variável cortisol no tempo "Após" em relação ao tempo "Antes". Análise por Oddis Ratio (OR) ou Razão de chances $(p<0,05)$

TABELA 4

Número de leucócitos (Mm3), Neutrófilos (mM3), Linfócitos (Mm3) e monócitos (mM3), nos tempos "ANtes" e "Após" A intervençÃo (N=9)

\begin{tabular}{lcccccrr}
\hline Variáveis & $\mathrm{X}$ & $\mathrm{dp}$ & $\mathrm{min}$ & máx & perc 25 & perc 50 & perc 75 \\
\hline Leucócitos Antes & 6888,9 & 1199,3 & 5300 & 8630 & 5720,0 & 6850 & 8040,0 \\
Leucócitos Após & $9585,6^{*}$ & 2017,6 & 7700 & 14090 & 7945,0 & 8950 & 10455,0 \\
Neutrófilos Antes & 3663,8 & 1371,3 & 1695 & 6472 & 2813,5 & 3300 & 4409,0 \\
Neutrófilos Após & $4683,9^{*}$ & 2038,7 & 2860 & 9300 & 3164,5 & 4157 & 5537,0 \\
Linfócitos Antes & 2080,3 & 627,2 & 1122 & 3080 & 1663,5 & 2034 & 2564,0 \\
Linfócitos Após & $3678,4^{*}$ & 809,4 & 2239 & 4664 & 3067,5 & 3922 & 4330,5 \\
Monócitos Antes & 769,7 & 187,1 & 530 & 1013 & 597,5 & 770 & 952,0 \\
Monócitos Após & $1209,8^{*}$ & 351,9 & 618 & 1656 & 861,5 & 1318 & 1465,5 \\
\hline
\end{tabular}

Os dados apresentados na tabela representam a média amostral $(X)$, desvio padrão (dp), valor mínimo (min), valor máximo (máx), percentil 25, percentil 50 e percentil 75. Média amostral (X), desvio padrão (dp), valor mínimo (min) e valor máximo (máx).

*Diferença estatisticamente significativa da concentração da variável no tempo "Após" em relação ao tempo "Antes". Análise por Oddis Ratio (OR) ou Razão de chances $(p<0,05)$

\section{Coletas sangüíneas}

Antes de iniciar a sessão de treinamento, os indivíduos foram submetidos a repouso absoluto durante 30 minutos, em seguida foi realizada a primeira coleta de sangue, onde foram coletados $20 \mathrm{ml}$ de sangue.

A mesma quantidade de sangue foi coletada imediatamente após o término da sessão de treinamento. Nos tempos de 24 e 48 h após o término da sessão de treinamento foram coletados $10 \mathrm{ml}$ de sangue.

Após as coletas, o sangue foi centrifugado por 10 minutos a $5000 \mathrm{~g}$ e armazenado a -200C.

As materiais utilizados na coleta eram todos descartáveis, etiquetados adequadamente e de reconhecida qualidade. A coleta do sangue foi realizada por um técnico habilitado e qualificado.

As análises foram realizadas no Laboratório de Análises Clínicas da Universidade Metodista de São Paulo (LABMESP).

\section{Determinação da concentração de creatina quinase (CK)}

Foram coletadas amostras para análise de CK ao início e término da sessão de treinamento, bem como 24 e 48h após a realização das mesmas. As amostras foram processadas no equipamento Cobas Mira S, utilizando-se a metodologia cinético-espectrofotométrica.

\section{Determinação da percepção subjetiva de dor}

Os sujeitos foram orientados a classificar a dor muscular "Antes", imediatamente "Após", "24h" e "48h" depois da sessão de treinamento. Foi entregue aos indivíduos uma folha com uma linha de $10 \mathrm{~cm}$, que representa uma escala de percepção subjetiva de dor. Nessa escala de análogos visuais (VAS, Visual Analog Scale) (Figura 1), uma das extremidades $(0 \mathrm{~cm})$ contém uma expressão verbal, "pouca dor" e a extremidade oposta (10 $\mathrm{cm})$ "muita dor"13. 
Os sujeitos assinalaram um local nessa linha que evidenciava a dor que eles estavam sentindo após "palpação" na região do peitoral maior, bíceps braquial e braquial, solicitados no treinamento. A distância em centímetros da extremidade $0 \mathrm{~cm}$ até o ponto indicado pelo sujeito foi medido com uma régua e considerado como a medida de dor.

\section{Análise Estatística}

Foram utilizados testes paramétricos e não paramétricos seguindo as recomendações de Diggle et al. ${ }^{14}$.

Para analisar dados dependentes em dois e quatro tempos foi adotado o método de equações de estimação generalizada (Generalized estimating equations - GEE), com distribuição gama e multinomial (não paramétrico).

Essa equação analisa os dados longitudinais em diferentes tipos de distribuição e compara entre os tempos ${ }^{14}$. Foi usado o pacote estatístico SPSS para Windows versão 11.0.

\section{RESULTADOS}

A média para a concentração sérica da variável testosterona na análise realizada "Antes" da intervenção foi 14,7 + 4,7 nmol/L e imediatamente "Após" foi 16,5 + 22,8 nmol/L (Tabela 3). Esses dados demonstram que a concentração de testosterona não se alterou com a intervenção, quando analisada por Oddis Ratio (OR) ou Razão de chances $(p=0,336)$.

A concentração sérica média de cortisol "Antes" da intervenção foi 343,2 + 115,5 nmol/L e "Após", 516,8 + 181,6 nmol/L (Tabela 3). Esses valores representam aumento significativo de $51 \%(p=0,001)$. A razão T/C não se alterou com a intervenção, e a média da razão T/C "Antes" da intervenção foi 0,048 + 0,026 e imediatamente "Após" a intervenção verificou-se 0,035 $+0,017$ de razão entre a concentração sérica de testosterona e cortisol (Tabela 3).

Houve aumento de $39 \%$ no número de leucócitos circulantes ( $p<0,05)$ "Após" a intervenção $(9585,6$ + 2017,6 mm3) em relação ao tempo "Antes" da intervenção $(6888,9+1199,3$ mm3) (Tabela 4). $\bigcirc$ número de neutrófilos circulantes foi $28 \%$ maior no tempo "Após" a intervenção $(4683,9$ + 2038,7 mm3) em relação ao valor encontrado "Antes" da realização do protocolo (3663,8 + 1371,3 mm3) (Tabela 4).

Os linfócitos foram as células que sofreram maior influência do protocolo adotado no presente estudo. Houve aumento de $77 \%$ do número de linfócitos circulantes no tempo "Após" a intervenção $(p<0,05)$. "Antes" de iniciar a sessão de treinamento a média foi de 2080,3 + 627,2 mm3, aumentando para 3678,4 + 809,4 mm3 imediatamente "Após" (Tabela 4).

Com relação ao número de monócitos, foi observado aumento de 57\% no tempo "Após" a intervenção $(p<0,05)$, quando comparado ao tempo "Antes" do treinamento $(769,7+187,1$ mm3 "Antes" e 1209,8 + 351,9 mm3 "Após") (Tabela 4).

\section{Discussão}

Este foi o primeiro estudo a avaliar as modificações nas respostas hormonais e imunes frente ao método de treinamento em bi-set, e também a manipulação das variáveis agudas do treinamento (alteração na ordem dos exercícios e manipulação no tipo e tempo de intervalo entre as séries).

treinamento de força pode modular as concentrações séricas do hormônio testosterona em homens ${ }^{6}$. As elevações encontradas na testosterona total podem ser atribuídas à redução do volume plasmático (influxo de fluídos para o sarcoplasma da célula muscular ativa), estimulação adrenérgica ${ }^{15}$, secreção estimulada pelo lactato $^{16}$ e adaptações potencializadas na síntese de testosterona e/ou capacidade secretória das células de Leydig ${ }^{17}$. No entanto, outros estudos não evidenciaram mudanças nas concentrações de testosterona ${ }^{7,18}$.

No presente trabalho, não foi encontrado mudança significativa na concentração sérica de testosterona total, nos indivíduos estudados (Tabela 3). De maneira similar, Ratamess et al. ${ }^{7}$ não encontraram alterações na testosterona circulante após um protocolo com 1 série de 10 repetições, a 80-85\% de 1 RM no exercício de agachamento.

Häkkinen e Pakarinen ${ }^{4}$ também não observaram alterações na testosterona total com 5 séries de 10 repetições no agachamento em mulheres. Ahtiainen et al. ${ }^{6}$ não registraram nenhuma mudança na resposta aguda da testosterona entre 2 diferentes protocolos de número similar de repetições, mas com intensidade ligeiramente maior em um dos protocolos.

Em contradição, Kraemer et al. ${ }^{19}$ registraram aumentos significativos na testosterona depois de protocolo com menor intensidade (50\% de 1 RM) e com 5 séries de 15 a 20 repetições no agachamento. Foi demonstrado que programas característicos do fisiculturismo (cargas moderadas e volumes altos) produzem maiores respostas na concentração de testosterona do que treinamentos mais intensos ${ }^{20}$.

Possivelmente, a duração reduzida da sessão de treinamento do presente estudo, 25 minutos no total, não tenha sido suficiente para alterar a resposta aguda deste hormônio, ao passo que, os trabalhos que encontraram alterações na testosterona utilizaram um período de duração superior ao atual (entre 45 minutos e 1 hora, em média) $)^{21,22}$.

O cortisol estimula a lipólise, aumenta a degradação protéica, e ainda reduz a síntese de proteínas. Devido ao seu importante papel na remodelação tecidual, alterações agudas e crônicas do cortisol durante o treinamento de força são freqüentemente examinadas ${ }^{8}$.

Uma sessão aguda de treinamento de força pode induzir elevações significativas do cortisol e do hormônio adrenocorticotrófico $(\mathrm{ACTH})^{18,21,23}$, com respostas similares entre homens e mulheres ${ }^{24}$. $O$ tempo de intervalo entre as séries parece influenciar a resposta ao cortisol.

No presente trabalho foi demonstrado aumento significativo de $51 \%$ (Tabela 3) na concentração do cortisol sérico frente à sessão de treinamento de força aplicada. Tendo em vista que, neste protocolo, o intervalo de recuperação foi ativo (com a realização de outro exercício de força, série combinada) e permaneceu no máximo por 1 minuto até que fosse realizada novamente uma outra série do mesmo exercício, pode-se sugerir que este intervalo foi suficientemente intenso para provocar as alterações observadas na concentração sérica de cortisol, como 
também foi detectado nos trabalhos acima que utilizaram entre 1 minuto e 1 minuto e meio de recuperação. Similarmente, Kraemer et al. ${ }^{3}$ registraram que a realização de 8 séries de 10RM no exercício leg press com 1 minuto de intervalo aumentou mais significativamente a resposta aguda do cortisol, do que o mesmo protocolo com período de descanso de 3 minutos.

Outro fator que pode ter conduzido ao aumento do cortisol no presente estudo foi o número de séries realizadas, 18 no total, e também o número de repetições, entre 8 e 12RMs em cada uma das séries. Estudos que utilizaram número de séries e repetições similares ao presente protocolo verificaram aumento na concentração de cortisol no plasma ${ }^{6,7,25}$. A razão entre testosterona e cortisol T/C tem sido utilizada para estabelecer as cargas de treinamento ${ }^{26}$ ou como indicativo de adaptação ou excesso de sobrecarga ${ }^{27}$.

A razão entre estes dois hormônios pode ser um parâmetro da relação de anabolismo/catabolismo muscular. Segundo Vervoorn et al. ${ }^{28}$ e Banfi et al. ${ }^{26}$ caso a diminuição da razão T/C seja maior que $30 \%$ o indivíduo pode se encontrar em uma situação de cansaço e/ou uma recuperação incompleta, caracterizando uma condição de catabolismo e prejudicando a resposta adaptativa aos treinamentos.

No presente estudo não foi encontrada diferença significativa na razão T/C (Tabela 3). O fato da testosterona não ter se alterado, pode ter sobreposto o aumento significativo do cortisol. Em função do aumento da intensidade e manutenção do volume do treinamento no atual estudo, é razoável hipotetizar que o volume do treinamento seja mais importante do que a intensidade para desencadear alteração na razão $T / C$, em praticantes de treinamento de força.

Em concordância, Banfi et al. ${ }^{26}$ investigaram o comportamento da razão $\mathrm{T} / \mathrm{C}$ em patinadores velocistas que treinavam intensamente e verificaram que o aumento abrupto no volume de treino foi o principal fator responsável por uma diminuição de 30\% na razão $\mathrm{T} / \mathrm{C}$, cronicamente. Uchida et al. ${ }^{27}$ não encontraram alteração na razão T/C após uma sessão de treinamento de força em mulheres treinadas, nesse caso não houve alteração na concentração de testosterona total, bem como de cortisol circulante.

O intervalo entre as séries foi de 90 segundos (intervalo passivo). No presente estudo, em função do método adotado (bi-set), provavelmente o estresse tenha sido maior, uma vez que os indivíduos terminavam um exercício e se dirigiam ao outro da combinação, executando-o sem intervalo passivo.

A leucocitose (aumento no número de leucócitos circulantes) ocorre em resposta a exercícios físicos intensos e de curta duração. Os efeitos deste tipo de exercício físico sobre o aumento no número de leucócitos circulantes são mediados, pelo menos em parte, pela ativação do sistema nervoso simpático ${ }^{29}$ e aumento agudo dos níveis séricos de catecolaminas durante o exercício ${ }^{30}$.

A leucocitose pode aumentar linearmente de acordo com a elevação da intensidade do exercício ${ }^{31}$. Uma maior secreção de catecolaminas pode ocorrer em sessões de treinamento de força que utilizam menores intervalos de recuperação $(<1$ minuto), quando comparadas a protocolos que utilizam períodos de recuperação maiores ( $>2$ minutos) $)^{24,32}$.

Outros mecanismos responsáveis pelo aumento na migração de leucócitos induzidos pelo exercício incluem: elevação da concentração de citocinas, mudanças na temperatura corporal, estado de hidratação e aumento no fluxo sangüíneo ${ }^{33}$.

Durante uma sessão aguda de treinamento de força, pode-se observar leucocitose, juntamente com linfocitose (aumento no número de linfócitos circulantes), monocitose (aumento no número de monócitos circulantes) e neutrocitose (aumento no número de neutrófilos circulantes) ${ }^{34,35,36}$. A leucocitose foi observada também num protocolo de força realizado no exercício leg press, com 8 séries de 10RMs a 70-90\% de 1RM, em sujeitos treinados em força ${ }^{3}$. $O$ protocolo de treinamento de força deste estudo induziu aumento agudo significativo de 39\% na contagem total de leucócitos, $28 \%$ nos neutrófilos e $77 \%$ nos linfócitos, após a sessão de treinamento de força (Tabela 4).

Mayhew et al. ${ }^{2}$ encontraram resultados semelhantes com indivíduos treinados em força que realizaram um protocolo com 10 séries de 10 repetições a 65\% de 1RM no exercício leg press utilizando um intervalo de recuperação de 1 minuto e 3 minutos. Estes pesquisadores observaram que intervalos de recuperação mais curtos (1 minuto) promoveram leucocitose mais pronunciada e maiores elevações nos linfócitos, monócitos e neutrófilos circulantes, quando comparados com intervalos mais prolongados (3 minutos).

As semelhanças entre os dois estudos podem estar relacionadas à intensidade utilizada, $70 \%$ neste estudo e 65\% no estudo de Mayhew et al. ${ }^{2}$, mas principalmente ao período de recuperação, praticamente o mesmo, de 1 minuto a 1 minuto e meio.

\section{CONSIDERAÇÕES FINAIS}

As alterações das variáveis agudas do treinamento de força como intervalo de recuperação entre as séries e o método de treinamento podem produzir modificações significativas nas células do sistema imune, promovendo o aumento nas concentrações circulantes de leucócitos totais, neutrófilos, linfócitos (em maior escala) e monócitos.

Também aumentam as concentrações circulantes de cortisol, indicando desta forma que o treinamento promoveu um estímulo diferenciado em indíviduos treinados. Estas respostas fisiológicas podem indicar a intensidade da carga interna de treinamento, visto que, a carga externa é comumente acompanhada pelos testes de força. As dosagens hormonais e imunológicas podem constituir-se numa importante ferramenta no acompanhamento das cargas do treinamento ao longo do ano, auxiliando na prevenção da síndrome do sobretreinamento e em sintomas de estresse devido a cargas excessivas.

O conhecimento das concentrações de testosterona, cortisol e o comportamento da razão T/C após uma sessão de treinamento podem auxiliar os treinadores, fisiologistas e educadores físicos na escolha do protocolo ideal para a população que busca potencializar a performance, a hipertrofia muscular e, sobretudo, melhorar a qualidade de vida.

Porém, o uso da razão T/C, assim como dos parâmetros imunes, como indicativos do estresse imposto ao organismo pelo treinamento de força exige cautela, uma vez que o seu comportamento ainda não está totalmente claro, necessitando de mais estudos para elucidar o assunto. 


\section{REFERÊNCIAS BIBLIOGRÁFICAS}

1. Potteiger JA, Chan MA, Haff GG. Training status influences T-cell responses in women following acute resistance exercise. J Strength Cond Res 2001;15:185-91.

2. Mayhew DL, Thyfault JP, Koch AJ. Rest-interval length affects leukocyte levels during heavy resistance exercise. J Strength Cond Res 2005; 19:16-22.

3. Kraemer WJ, Clemson A, Triplett NT, Bush JA, Newton RU, Lynch $J M$. The effects of plasma cortisol elevation on total and differential leukocyte counts in response to heavy-resistance exercise. Eur J Appl Physiol Occup Physiol 1996;73:93-7.

4. Häkkinen K, Pakarinen A. Acute hormonal responses to heavy resistance exercise in men and women at different ages. Int J Sports Med 1995; 16:507-13.

5. Schwab R, Johnson GO, Housh TJ, Kinder JE, Weir JP. Acute effects of different intensities of weight lifting on serum testosterone. Med Sci Sports Exerc 1993;25:1381-5.

6. Ahtiainen JP, Pakarinen A, Kraemer WJ, Häkkinen K. Acute hormonal and neuromuscular responses and recovery to forced vs maximum repetitions multiple resistance exercises. Int J Sports Med $2003 ; 24: 410-8$

7. Ratamess NA, Kraemer WJ, Volek JS, Maresh CM, Vanheest JL, Sharman MJ, et al. Effects of heavy resistance exercise volume on post-exercise androgen receptor content in resistance-trained men. J Steroid Biochem Mol Biol 2005;93:35-42.

8. Kraemer WJ, Ratamess NA. Hormonal responses and adaptations to resistance exercise and training. Sports Med 2005;35:339-61.

9. American College of Sports Medicine (ACSM) and Dietitians Canada Joint Position Statement. Nutrition and Athletic Performance. Med Sci Sports Exerc 2000;32:2130-45.

10. Matuszak ME, Fry AC, Weiss LW, Ireland TR, Mcknight MM. Effect of rest interval length on repeated 1 repetition maximum back squats. J Strength Cond Res 2003;17:634-7.

11. Brown LE, Weir JP. Procedures Recommendation I: Accurate Assessment Of Muscular Strength And Power. J Exerc Physiol 2001;4:1-21.

12. American College of Sports Medicine (ACSM). Progression Models in Resistance Training for Healthy Adults. Med Sci Sports Exerc 2002;34:364-80.

13. Clarkson PM, Hubal MJ. Exercise-induce Muscle Damage in Humans. Am J Phys Rehabil 2002;81:S52-69.

14. Diggle PT, Liang KY, Zeger SL. Analysis of longitudinal data. New York: Oxford University Press; 1994.

15. Jezova $D$, Vigas $M$. Testosterone response to exercise during blockade and stimulation of adrenergic receptors in man. Horm Res $1981 ; 15: 141-7$.

16. Lin H, Wang SW, Wang RY, Wang PS. Stimulatory effect of lactate on testosterone production by rat Leydig cells. J Cell Biochem $2001 ; 83: 147-54$

17. Fry AC, Kraemer W.J. Resistance exercise overtraining and overreaching: neuroendócrino responses. Sports Med 1997;23:106-29.

18. Häkkinen K, Pakarinen A, Alen M, Kauhanen H, Komi PV. Neuromuscular and hormonal responses in elite athletes to two successive strength training sessions in one day. Eur J Appl Physiol Occup Physiol $1988 ; 57: 133-9$

19. Kraemer WJ, Volek JS, French DN, Rubin MR, Sharman MJ, Gomez $A L$, et al. The effects of L-carnitine L-tartrate supplementation on hormonal responses to resistance exercise and recovery. J Strength Cond Res 2003;17:455-62.

20. Kraemer WJ, Gordon SE, Fleck SJ, Marchitelli LJ, Mello R, Dziados $\mathrm{JE}$, et al. Endogenous anabolic hormonal and growth factor responses to heavy resistance exercise in males and females. Int J Sports Med $1991 ; 12: 228-35$.

21. Kraemer WJ, Häkkinen K, Newton RU, Nindl BC, Volek JS, McCormick $M$, et al. Effects of heavy resistance training on hormonal response patterns in younger vs older men. J Appl Physiol 1999;87:982-92.

22. Tremblay MS, Copeland JL, Van Helder W. Effect of training status and exercise mode on endogenous steroid hormones in men. J Appl Physiol 2003;96:531-9.

23. Guezennec Y, Leger L, Lhoste F, Aymonod M, Pesquies PC. Hormone and metabolite response to weight-lifting training sessions. Int J Sports Med 1986;7:100-5.

24. Kraemer WJ, Fleck SJ, Dziados JE, Harman EA, Marchitelli LJ, Gordon SE, et al. Changes in hormonal concentrations after heavyresistance exercise protocols in women. J Appl Physiol 1993;75:594604.

25. Smilios I, Pilianidis T, Karamouzis M, Tokmakidis SP. Hormonal responses after after various resistance exercise protocols. Med Sci Sports Exerc 2003;35:644-54.

26. Banfi G, Marinelli M, Roi GS, Agape V. Usefullness of free testosterone/ cortisol ratio during season of elite espeed skating athletes. Int J Sports Med 1993;14:373-9.

27. Uchida MC, Bacurau RFP, Navarro F, Pontes LF, Tessuti VD, Moreau RL, et al. Alteração da relação testosterona:cortisol induzida pelo treinamento de força em mulheres. Rev Bras Med Esporte 2004; 10:165-8

28. Vervoorn C, Quist AM, Versmulst LJM, Erich WB, De Vries WR, Thijssen JH. The behavior of the plasma free testosterone/cortisol ratio during off season of elite rowing training. Int J Sports Med $1991 ; 12: 257-63$.

29. Mills PJ, Rehman J, Ziegler MG, Carter SM, Dimsdale JE, Maisel AS. Nonselective beta blockade attenuates the recruitment of CD62L(IT lymphocytes following exercise. Eur J Appl Physiol Occup Physiol 1999;79:531-4.

30. Mazzeo RS. Catecholamine responses to acute and chronic exercise. Med Sci Sports Exerc 1991;23:839-45.

31. Bain BJ, Phillips D, Thomson K, Richardson D, Gabriel I. Investigation of the effect of marathon running on leucocyte counts of subjects of different ethnic origins: relevance to the aetiology of ethnic neutropenia. British J Haematol 2000;108:483-7.

32. Kraemer WJ, Noble BJ, Clark MJ, Culver BW. Physiologic responses to heavy resistance exercise with very short rest periods. Int J Sports Med 1987;8:247-52.

33. Nieman DC. Immune response to heavy exertion. J Appl Physiol 1997;82:1385-94

34. Flynn MG, Fahlman M, Braun WA, Lambert CP, Bouillon LE, Brolinson $P G$, et al. Effects of resistance training on selected indexes of immune function in eldery women. J Appl Physiol 1999;86:1905-13.

35. Miles MP, Leach SK, Kraemer WJ, Dohi K, Bush JA, Mastro AM. Leukocyte adhesion molecule expression during intense resistance exercise. J Appl Physiol 1998;84:1604-9.

36. Nieman DC, Henson DA, Sampson CS, Herring JL, Suttles J, Conley $M$, et al. The acute immune response to exhaustive resistance exercise. Int J Sports Med 1995; 16:322-8. 